\title{
Characterization of LoRa Point-to-Point Path-Loss: Measurement Campaigns and Modeling Considering Censored Data
}

\author{
Gilles Callebaut and Liesbet Van der Perre
}

\begin{abstract}
Low-Power Wide-Area technologies have demonstrated their usefulness in a wide variety of Internet of Things applications. New applications are emerging, requiring a mesh or point-to-point topology, in contrast to the conventional star-ofstars topology. In this paper, we evaluate the coverage and model the path loss of these links, based on experimental campaigns in three environments: urban, forest and coastal. More obstructions, scattering and diffraction are encountered because the terminals are typically at a low height. This results in a higher path loss compared to the star-of-stars topology. Consequently, more packets drop below the receiver sensitivity. Realistic path loss parameters are estimated by also taking the censored data into account. The packet error ratio is determined based on the estimated path loss parameters to evaluate the performance of point-to-point links. Even in these adverse environments, $80 \%$ of the transmitted packets are successfully received at a distance of approximately $200 \mathrm{~m}$. Moreover, a range of over $4 \mathrm{~km}$ is observed in the line-of-sight scenario. Despite the unfavorable radio propagation in the urban scenario and the densely forested terrain, a maximum range of $1 \mathrm{~km}$ is achieved.
\end{abstract}

Index Terms-LPWAN, LoRa, Point-to-Point, Mesh, Path loss model, censored data, IoT

\section{INTRODUCTION}

$\mathbf{I}$ NTERNET-OF-THINGS applications are facilitated in diverse domains through the advances in low-cost sensors and wireless communication technology. However, deployments face persistent challenges: coverage for many Internet of Things (IoT) networks is not omnipresent, costs for network infrastructure and subscription are undesirable and energy consumption at the node needs to be reduced to increase battery life. For the latter, the radio often plays a dominant role.

Long-Range Wide-Area Network (LoRaWAN) is a suitable communication technology to connect IoT applications requiring medium to long range connectivity [1] and hence it is considered by many IoT applications to relay their telemetry data to a back-end system. Despite the high number of deployments of these networks, IoT nodes, being often positioned in remote locations, still lack coverage. In addition, the accompanied subscription cost possibly results in a too high expenditure

Manuscript received June 14, 2019; revised September 14, 2019; accepted November 10, 2019. This research was partially supported by the IoTRAILER Use Case project funded by the EU Horizon 2020 program IoF2020 under under Grant No 731884. (Corresponding author: Gilles Callebaut)

The authors are with the Department of Electrical Engineering, KU Leuven, DRAMCO, B-9000 Ghent, Belgium (gilles.callebaut@kuleuven.be).

Copyright (c) 2019 IEEE. Personal use of this material is permitted. However, permission to use this material for any other purposes must be obtained from the IEEE by sending a request to pubs-permissions@ieee.org per node. Actually, many applications do not require extensive antenna and gateway infrastructure. A LoRa point-to-point system facilitates an easy deployment. The entry-barrier to set up point-to-point links is particularly low as neither a license, nor significant infrastructure investment is required. It opens opportunities to establish 'Ad-hoc-Networks-of-Things' with considerable coverage, which can be private if desired. Potential application domains include large farms in remote locations, ports, nature reserves. Moreover, these networks can be easily relocated, as illustrated in Figure 1
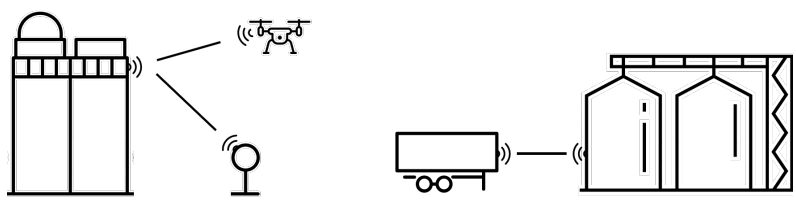

Fig. 1: Left: star network topology with the gateway positioned at height. Right: P2P ad-hoc setup. It demonstrates (1) the additional required communication between the gateway and the back-end and (2) the high placement of the gateway in conventional star networks. By adopting a point-to-point network, the IoT nodes can be grealty simplified, addressing new use case requirements.

In this paper, we address the main challenge for point-topoint (P2P) LoRa installations, i.e., the unclear connectivity range. Several authors have reported on empirical studies of the coverage for centrally operated LoRa networks, i.e. LoRaWAN, where the access points typically are placed at an elevated location [2, 3, 4, 5, 6, 7, 8, 9]. Also indoor deployments have been considered in papers, where clearly a long range is not targeted [10]. Outdoor-to-indoor propagation is investigated in [11] where a neural network is applied to determine a path loss (PL) model. In this work, we evaluate the PL on a Long Range (LoRa) link in a point-to-point setting with low-height terminals $(1.5 \mathrm{~m})$ and operating at $868 \mathrm{MHz}$. We hence investigate which range can be expected in outdoor deployments, focusing on the observed propagation channel by the LoRa nodes.

We have performed four measurement campaigns to acquire representative coverage data for the vast diversity of the environments where these systems can be deployed. The measurements have been conducted with actual LoRa devices, yielding results which include the behavior of the radio elements; in contrast to employing a channel sounder. Several authors have 
identified the need to consider censored data for reliable PL modeling [12, 13, 14]. Their work is particularly relevant for the analysis in this paper. The resulting model can support radio planning and application performance assessment for a variety of IoT services.

Contribution. The contribution of this paper is threefold. First, we build a portable measurement set-up and made it available in open source. Experimental campaigns in three different environments were performed with this set-up. Secondly, path loss models are fitted based on the measured censored data in realistic environments with non-uniform numbers of samples with respect to the distance. Finally, the packet error ratio (PER) for P2P LoRa links is determined to evaluate the link performance. The PL model derived in the urban scenario is evaluated based on other empirical path loss models and a measurement campaign conducted in another city. Both the path loss models and the PER can be adopted in network planning and in simulations to design new MAC protocols. A few illustrative examples of successful P2P LoRa deployments are provided.

This paper is further organized as follows. LoRa P2P systems are introduced in Section III with a focus on the difference between typical star-of-star networks and P2P and mesh networks adopting the LoRa technology. Next, in Section III. we present the in-house developed experimental setup which has been made available in open source [15, 16, 17], and we describe the measurement procedure. Section IV focuses on the path loss modelling approach accounting for censored measured data, in urban, coastal and densely forested environments. We present and discuss the main modeling results in Section V. Section VI presents illustrative examples where P2P LoRa links can provide the desired connectivity. Finally in section VII the main conclusions of this paper are summarized.

\section{LoRA POINT-TO-PoInT SPECIFICATION}

LoRa achieves long-range connectivity by exploiting a coding gain originating from the spread spectrum modulation technique. More specifically, chirp spread spectrum (CSS) is employed to modulate symbols over a fixed bandwidth using chirps. The spreading factor (SF) determines the amount of chirps. The range can be extended by increasing the SF. The associated gains for each spreading factor are summarized in Table I By increasing the SF, the data rate is reduced and the time on air is increased, causing a higher energy consumption. Hence, the LoRa modulation technique enables a trade-off between range and energy consumption by adapting the SF. The LoRaWAN protocol specifies the medium access control (MAC) layer and the topology of the network. In this work, we focus on systems employing only the LoRa PHY layer to create $\mathrm{P} 2 \mathrm{P}$ connectivity, offering advantages over utilizing the LoRaWAN specification [1] for many use cases. Figure [1 illustrates the distinction between a LoRaWAN network and the proposed LoRa P2P architecture. The P2P setup consists of low-cost hardware in contrast to a more expensive gateway in the LoRaWAN network.

In its simplest form, the devices can be configured to send-andforget, allowing for straightforward setup if the application is already developed; yielding power improvements and lower device expenditure. Moreover, devices are not mandated to be registered to a network prior to sending packets, contrary to a deployment in a LoRaWAN. Additionally, edge computing techniques [19] can be easily implemented at the concentrator or nodes, because no back-end connection is required. This in contrast to LoRaWAN, where the telemetry data is always aggregated by the receiving gateways and forwarded to the cloud. Edge computing is not an option due to the design of the security of the LoRaWAN protocol.

LoRa P2P devices can exploit band G3 (Table II) otherwise used by LoRaWAN gateways for downlink communication. The high duty cycle and a higher allowed transmit power can be favorable over the other available bands 1 Furthermore, LoRaWAN gateways make use of IQ inversion (i.e., LoRa Modulation Polarity Inversion) to mitigate interference between uplink and downlink communication. The IQ inversion changes the sign of the slope of the frequency-time function, i.e., upchirps become downchirps. As a consequence, P2P devices operating in band G3 are subjected to less interference in the presence of LoRaWAN networks. Evaluations of a traditional star topology LoRa in terms of coverage cannot be readily applied to LoRa $\mathrm{P} 2 \mathrm{P}$ links because of the above reasons and moreover, and not in the least, because of the high placement of gateways (several meters). For a smooth installation of P2P links one prefers to keep the nodes at hand's reach. We here assumed $1.5 \mathrm{~m}$ to be a convenient height.

One can anticipate to extend the arrangement of LoRa P2P links to a mesh network, offering similar advantages in terms of ease of deployment and flexibility. However, the adoption of LoRa in a mesh network poses additional challenges. Lundell

${ }^{1}$ To be complete, one can also exceed the permitted airtime, limited by the duty cycle, by applying listen before talk (LBT) and adaptive frequency agility (AFA). This will result in a higher energy consumption.

TABLE I: Sensitivity of the LoRa demodulator (BW125 kHz) expressed in received signal strength (RSS) and signal-to-noise ratio (SNR) for the used LoRa chip [18].

\begin{tabular}{lcc}
\hline Spreading Factor & RSS $(\mathbf{d B m})$ & SNR $(\mathbf{d B})$ \\
\hline SF6 & -118 & -5 \\
SF7 & -123 & -7.5 \\
SF8 & -126 & -10 \\
SF9 & -129 & -12.5 \\
SF10 & -132 & -15 \\
SF11 & -133 & -17.5 \\
SF12 & -136 & -20 \\
\hline
\end{tabular}

TABLE II: LoRaWAN bands According to the ETSI Regulations

\begin{tabular}{|c|c|c|c|c|}
\hline \multirow[t]{2}{*}{ Band } & \multirow{2}{*}{$\begin{array}{l}\text { Frequency } \\
\mathrm{MHz}\end{array}$} & \multirow{2}{*}{$\begin{array}{l}\text { Duty } \\
\text { Cycle } \\
\%\end{array}$} & \multicolumn{2}{|c|}{ Max ERP } \\
\hline & & & $\mathrm{mW}$ & $\mathrm{dBm}$ \\
\hline g1 & $868.0-868.6$ & 1 & 25 & 14 \\
\hline g2 & $868.7-869.2$ & 0.1 & 25 & 14 \\
\hline g3 & $869.4-869.65$ & 10 & 500 & 27 \\
\hline g4 & $869.7-870$ & 1 & 25 & 14 \\
\hline
\end{tabular}


et al. [20] have presented a proof of concept of multi-hop routing in LoRa. LoRa is limited by its maximum payload size and its duty cycle due to the band it operates in; limiting the scalability of such networks. Furthermore, the receive windows present in the LoRaWAN specification can result in unwanted delays when relaying data between LoRaWAN nodes. Also, the additional overhead of the mesh protocol needs to be minimized because of power constrictions. To overcome the duty cycle limitations and mitigate interference, the protocol needs to consider multichannel operation. The protocol can hence be tailored to the application and the topology of the network. This allows to optimize for energy efficiency, by for instance reducing (redundant) on-time, and establish suitable connectivity based on an easy LoRa mesh network.

\section{Measurement Setup And Procedure}

We have realized an experimental setup with low-height transmitters and receivers, representative for use cases where low complexity antenna and gateway installations are required. Multiple experimental campaigns have been conducted to study the radio propagation of the LoRa physical layer. A platform, consisting of a LoRa modem [18], has been developed. The hardware [15] -hosting an SX1276 [18] as well as the software [16] is open-source to enhance the reproducibility of the experimental results. Furthermore, the raw measurement data and the processing files [17] are also readily available to encourage researchers to validate our results and allow extending our work.

We determine the actual $\mathrm{Rx}-\mathrm{Tx}$ separation distance based on the GPS coordinates of each module. A stationary setup is established at the $\mathrm{Tx}$ side comprising three transceivers programmed to transmit packets at different spreading factors. The Rx is instantiated based on a hand-held device, illustrated in Figure 2a The mobile receivers collect the radio frequency (RF) parameters (i.e., RSS and SNR) of each received packet. The transmitters are positioned on a tripod, as shown in Figure $2 \mathrm{~b}$. The devices are at a height of approximately $1.5 \mathrm{~m}$ above ground and spaced at a distance of $2 \lambda$ relative to each other to mitigate antenna coupling. We opted for a height of $1.5 \mathrm{~m}$ making the nodes easily accessible by humans as is the case for multiple IoT applications [21, 22] $]^{2}$ The three transmitters have each been set to transmit at a fixed SF, respectively SF7, SF9 and SF12. As introduced in Section II. the data rate depends on the spreading factor. Thus, the maximum data rate is obtained with SF7, while SF12 results in the minimum data rate. In this manner, we are able to collect a large number of samples at SF7 while also achieving the maximum range as a result of transmitting at SF12. The transmit output power is set at the highest level supported by the transceiver, i.e., $20 \mathrm{dBm}$, on all three devices.

Mobile receivers equipped with two types of antennas have been utilized in the measurement campaigns: either a quarter wave helical antenna [23] or a PCB antenna [24]. Both antennas feature a quasi-omnidirectional radiation pattern with

\footnotetext{
${ }^{2}$ Note, that the range can be further extended by increasing the height of both nodes and creating a line-of-sight (LoS) connection. By doing so, we were able to receive packets at a distance of over $40 \mathrm{~km}^{3}$
}

a gain of $2.15 \mathrm{dBi}-3 \mathrm{dBi}$ and an efficiency of $-2.97 \mathrm{~dB}$ at $868 \mathrm{MHz}$. Each time a packet is received, it is logged with the corresponding position.

All receivers are able to receive packets on all three spreading factors. Each receiver starts listening on the lowest LoRa spreading factor: SF7. The receivers increase their spreading factor if they do not longer receive packets, similar to the adaptive data rate (ADR) scheme adopted in LoRaWAN. Data points with inaccurate GPS coordinates -based on the delusion of precision- are discarded from the measurements in postprocessing.

We have conducted four measurement campaigns in three distinct environments, i.e., urban, coastal, and forest. Based on three initial campaigns the PL models were derived. The fourth measurement campaign serves as a test data set to assess the PL model. The campus of our university in the city of Ghent and surrounding area has been used as a representative case of an urban scenario. A coastal environment (the beach in Nieuwpoort, Belgium) has been chosen to illustrate a LoS scenario, while a forest (Het Leen, Eeklo) constitutes a densely vegetated environment. The characteristics of the experimental data collections for each scenario is summarized in Table III The fourth measurement was carried out in the city of Leuven and the obtained data was used as a test set for the derived urban model.

\section{PATH Loss Analysis And Modelling}

\section{A. Path Loss Models}

PL models predict the reduction in power of a signal as it propagates through a medium. They include effects such as the expansion of the radio wave and reflection, scattering, diffraction and absorption. Path loss is affected by the environment, the transmitter and receiver separation distance and the height of the transmit and receive antennas. Therefor, the PL model parameters are typically only valid for a particular frequency range, antenna arrangement, and environment. For this reason, this work characterizes LoRa P2P links in three environments. Path loss is conventionally modeled using a log-distance power law with a random term [25]. The log-distance path loss model in $\mathrm{dB}$ is expressed as,

$$
P L_{m}(d)=P L\left(d_{0}\right)+10 n \log _{10}\left(\frac{d}{d_{0}}\right)+\chi_{\sigma}, d \geq d_{0},
$$

where $d$ is the distance between receiver and transmitter in meters, $n$ the path loss exponent, $P L\left(d_{0}\right)$ is the path loss at a reference distance of $d_{0}$ and $\chi_{\sigma}$ describes the variation around the mean large-scale fading which is modeled as $\mathcal{N}\left(0, \sigma^{2}\right)$. We have considered a reference distance $d_{0}$ of $1 \mathrm{~m}$ for the modeling experiments. The $P L\left(d_{0}\right)$ is estimated as a result of the fitting method on the measured data. We let the subscript $m$ denote the modeled PL while using $s$ for the sampled (or observed) PL, i.e., $P L_{s}\left(d_{i}\right)$. The standard deviation $\sigma$ can be modelled either as a scalar (i.e. constant) or a distancedependent variable,

$$
\sigma(d)=a \log _{10}\left(\frac{d}{d_{0}}\right)+b .
$$




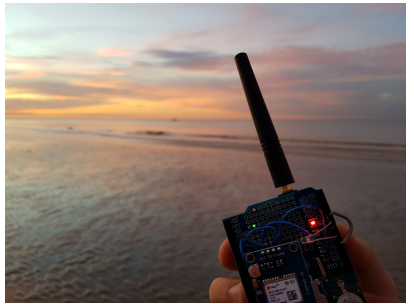

(a) Receive side implemented on a hand-held device to carry out the measurement campaigns.

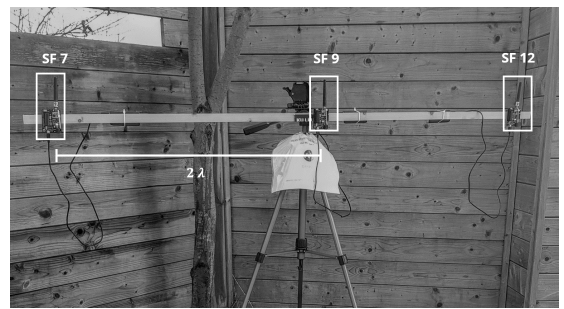

(b) Transmit side with three transmitters, equally spaced $(2 \lambda)$.

\begin{tabular}{llcl}
\hline & Tx 1 & Tx 2 & Tx 3 \\
\hline Tx Transmit Power & \multicolumn{3}{c}{$20 \mathrm{dBm}$} \\
Tx Spreading Factor & SF7 & SF9 & SF12 \\
Transmit Time Interval & $0.5 \mathrm{~s}$ & $1 \mathrm{~s}$ & $4 \mathrm{~s}$ \\
Tx/Rx Height & \multicolumn{3}{c}{$1.5 \mathrm{~m}$} \\
Frequency Band & \multicolumn{3}{c}{$869.525 \mathrm{MHz}(\mathrm{g} 3)$} \\
\hline
\end{tabular}

(c) System Parameters.

Fig. 2: Measurement setup with receivers (a) and transmitters (b) and system parameters (c). Both transmitters and receivers are placed at a height of approximately $1.5 \mathrm{~m}$.

Besides a single slope model, the PL can also be modeled by a dual slope as expressed in Eq. 3. This model is most appropriate in scenarios where two components are predominantly present at the receiver, i.e., a single (ground) reflected and a line-of-sight signal. In this model, the path loss exponent is equal to the free-space exponent up to a breaking distance $d_{c}$, whereafter the path loss increases proportional to $d^{4}$ due to the destructive interference of the LoS and the reflected ray. The breaking distance can be approximated by,

$$
d_{c}=\frac{4 h_{t} h_{r}}{\lambda}
$$

with $h_{t}$ and $h_{r}$ the height of the $\mathrm{Tx}$ and $\mathrm{Rx}$ antennas respectively.

Besides the log-distance path loss model, the empirical model obtained in [9], the Okumura Hata Model and the COST Hata model [25] are used to evaluate our PL models. Note, that most existing models expect the base station to be put higher than the $1.5 \mathrm{~m}$, advocating the need for an empirical PL model for LoRa P2P links with low-height terminals.

\section{B. Weighted Fitting}

We determine the PL parameters to best fit the experimental results, with weighting on the measurements applied to avoid that the distances with a high number of samples have an excessive impact on the fitting. The density plot of the observations for the urban environment, shown in Figure 3 . demonstrates the non-uniform density of the distribution of data points.

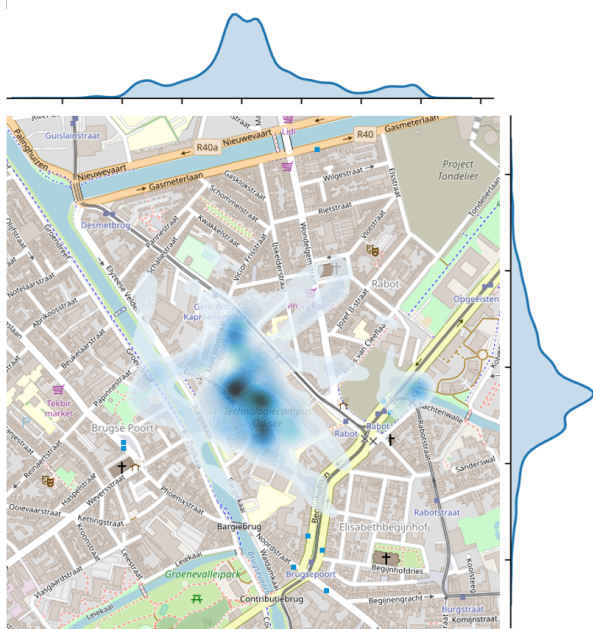

Fig. 3: Density of observations in the urban environment illustrating the non-uniform geographical distribution. This raises the need for a weighted fitting.

We account for this effect by attributing appropriate weights based on the distance of the samples. To that end, we categorize the measurement samples in bins. The weight $w$ for each sample is inversely proportional to the number of samples in its bin $N_{b}$,

$$
w=\frac{1}{N_{b}} \frac{N_{s}}{N},
$$

where $N$ is the number of bins and $N_{s}$ is the total number of samples. We chose the number of bins in such a way that they represent ring-shaped zones with equal difference of distances of $20 \mathrm{~m}$; between the outer and inner radius. We evaluate four weighting approaches on the bins [14]:

a) Equal weighting to each sample: This method is equivalent to not giving weights to the individual samples. It favors distances where more samples are present.

b) Equal weighting over distance d: Samples in bins with a smaller number of samples, w.r.t. the average, are given more weight.

c) Equal weighting over distance $\log _{10}(d)$ : By utilizing equal-size bins over $\log _{10}(d)$, shorter distances are favored over larger distances. 


$$
P L_{m}(d)= \begin{cases}P L\left(d_{0}\right)+10 n_{1} \log _{10}\left(\frac{d}{d_{0}}\right), & \text { if } d<d_{c} \\ P L\left(d_{0}\right)+10 n_{1} \log _{10}\left(\frac{d_{c}}{d_{0}}\right)+10 n_{2} \log _{10}\left(\frac{d}{d_{c}}\right), & \text { if } d \geq d_{c}\end{cases}
$$

d) Equal weighting over distance $d^{2}$ : PL samples at larger distances have a bigger impact than shorter distances, opposed to weighting method c).

We set all weights in bins with less than $5 \%$ of the average number of samples per bin to $w\left(d_{i}\right)=1$ to not overfit to underrepresented distances.

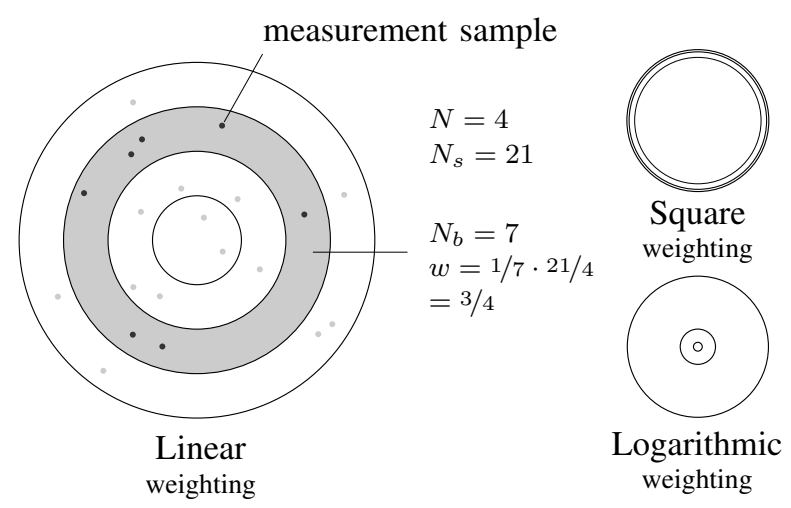

Fig. 4: Illustration of the utilized weighting approaches. The logarithmic weighting will favor larger distances, while the square distance method will increase the weight at short RxTx separation distances.

\section{Modelling with censored measurement data}

Our analysis targets the characterization of LoRa point-topoint PL and the experimental campaigns have been performed with actual transmitters and receivers. This approach, hence, yields measurements for the observed propagation channel, which includes the responses of the transmitter and receiver. In LoRa, the receiver sensitivity depends on the employed spreading factor. Increasing the SF lowers the demodulation floor, thereby extending the coverage or, equivalently, increasing the dynamic range of the PL measurements. The minimum RSS and SNR for each spreading factor are summarized in Table 1 Due to the limited receiver sensitivity, a number of packets can not be demodulated and thus they are not observed. As a result, the path loss data points are censored, thereby truncating the series of data samples as can be seen in Figure 5.

Conventionally, the ordinary least squares (OLS) method is utilized to estimate the PL parameters. However, this approach yields biased results as a consequence of neglecting the truncated samples. In order to account for the biased measurements, Gustafson et al. [26] propose using a Tobit model [27] and estimating the path loss parameters with a maximum-likelihood (ML) model. The latter method assumes that the observations follow a censored normal distribution. We adopt this approach and include the censored data points for which we only know the Tx-Rx separation distance. In doing so, we also take into account the unobserved measurement samples. The observations are considered to be censored when the path loss exceeds a threshold $P L_{t h}$. This threshold depends on the spreading factor applied on a specific LoRa packet. The demodulation floor of the RSS (Table I) is used to determine the $P L_{t h}$ for each packet.

As the model assumes that the observations follow a censored normal distribution, the probability of observing a censored data point at a distance $d$ is given by,

$$
\operatorname{Pr}\left(P L_{s}>P L_{\mathrm{th}}(\mathrm{SF})\right)=1-\Phi\left(\frac{P L_{\mathrm{th}}(\mathrm{SF})-P L_{m}(d)}{\sigma}\right)
$$

where $\Phi$ is the cumulative distribution function (CDF) of the standard normal distribution. Consequently, we expect that if a value is censored that the sample is situated in the upper tail of the probability density function (PDF) of the standard normal distribution. The PDF of the uncensored measurements is used for estimating the likelihood of observing an uncensored sample. Hence, the likelihood of a measurement, performed at distance $d_{i}$, either belonging to the observed or to the censored data can be expressed as [27],

$$
\mathrm{LF}= \begin{cases}\frac{1}{\sigma} \phi_{u}\left(\frac{\mathrm{PL}_{s}\left(d_{i}\right)-\mathrm{PL}_{m}\left(d_{i}\right)}{\sigma\left(d_{i}\right)}\right), & \text { if } P L_{s}\left(d_{i}\right) \leq \\ 1-\Phi\left(\frac{P L_{\mathrm{th}}(\mathrm{SF})-\mathrm{PL}_{m}\left(d_{i}\right)}{\sigma\left(d_{i}\right)}\right), & P L_{\mathrm{th}}(S F) \\ 1-\text { otherwise }\end{cases}
$$

where $(\phi)$ denotes the PDF of the standard normal distribution. By introducing an indicator function $I$,

$$
I= \begin{cases}1, & \text { if } \mathrm{PL}_{s}\left(d_{i}\right) \leq P L_{\text {th }} \\ 0, & \text { otherwise }\end{cases}
$$

we can write the log-likelihood function as,

$$
\begin{aligned}
& L=\sum_{i=1}^{N_{s}} I_{i} {\left[-\ln \left(\sigma\left(d_{i}\right)\right)+\ln \phi_{u}\left(\frac{\mathrm{PL}_{s}\left(d_{i}\right)-\mathrm{PL}_{m}\left(d_{i}\right)}{\sigma\left(d_{i}\right)}\right)\right] } \\
&+\sum_{i=1}^{N_{s}}\left(1-I_{i}\right) \ln \left[1-\Phi\left(\frac{P L_{\mathrm{th}}-\mathrm{PL}_{m}\left(d_{i}\right)}{\sigma\left(d_{i}\right)}\right)\right] .
\end{aligned}
$$

We estimate the path loss parameters by maximizing this likelihood function, i.e., $\hat{P L}\left(d_{0}\right), \hat{n}$ and $\hat{\sigma}$. Note, that without censored data points this likelihood function will give the same result as the ordinary least squares method.

\section{Modeling Results And InTERPREtation}

The results of the OLS and ML estimation including censored data for the three distinct environments are shown in Table IV 

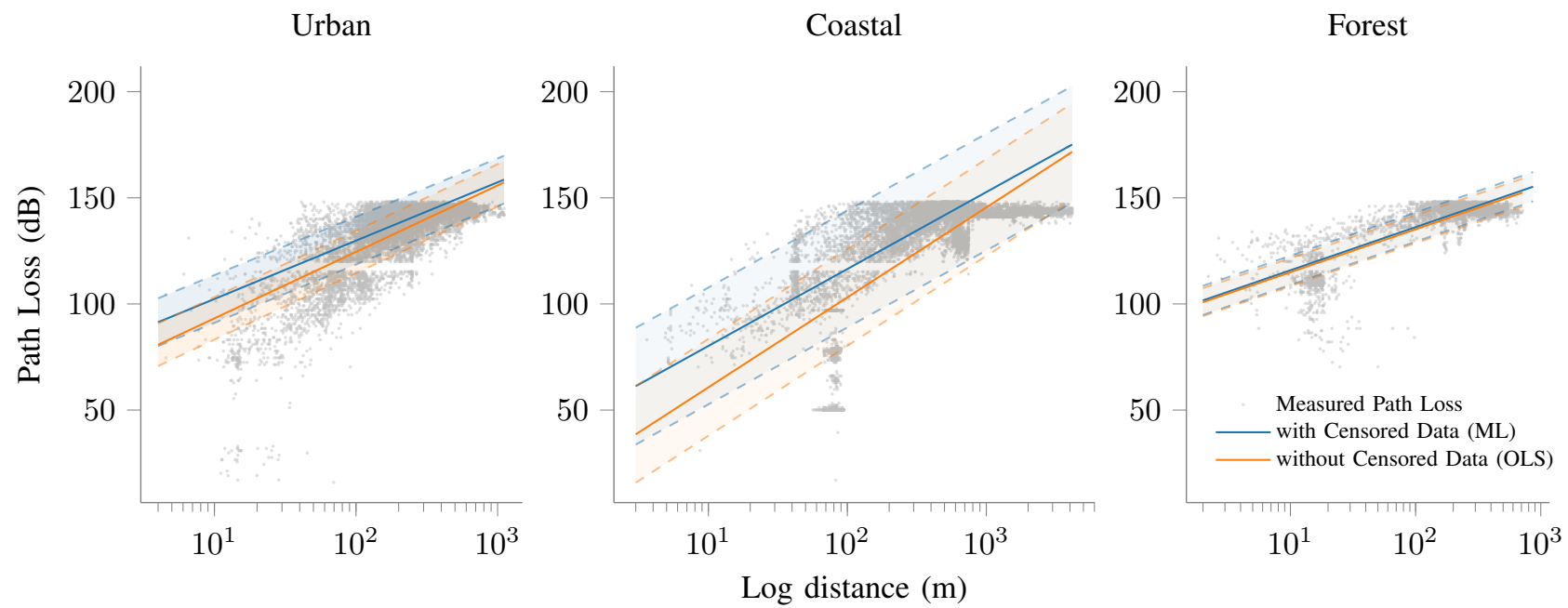

Fig. 5: Measured data of path loss versus distance. The censoring of data caused by the receiver sensitivity threshold can be observed.

TABLE III: Characteristics of the experimental data collections.

\begin{tabular}{llll}
\hline Environment & Max. distance $(\mathrm{m})$ & No. received packets & censored (\%) \\
\hline Urban (Ghent) & 1112 & 10947 & 40 \\
Urban (Leuven) $^{*}$ & 1036 & 11916 & - \\
Coastal & 4154 & 11017 & 30 \\
Forested & 716 & 4266 & 19 \\
\hline
\end{tabular}

* Measurement set is used to evaluate the predicted urban PL model based on the samples collected in the city of Ghent.

These path loss parameters are for a single slope (1) model with no weighting applied on the data. We assume that the antenna gain and the small-scale fading do not influence the fitted model given the high number of measurement samples collectively average out these aspects.

The resulting models are shown together with the experimental data in Figure 5. These demonstrate that LoRa nodes permit to establish P2P links exceeding $100 \mathrm{~m}$, even up to several kilometers, with terminals placed at a low height. We observe that the ML modelling method yields more realistic results, in line with the conclusions in [13]. Especially, the OLS underestimates the variance on the large-scale fading.

Further verification of the model was carried out based on a follow up experimental campaign in the city Leuven. The obtained urban model and other empirical models together with the measurements are depicted in Figure 7. It can be observed that the Okumura Hata model greatly overestimates the path loss, while the adapted COST Hata model and the models obtained by Petajajarvi et al. [9] underestimate the path loss. The Root Mean Square Error (RMSE) and Mean Absolute Error (MAE), observable in Table V] confirm that the derived path loss model presented in this paper, based on ML, is the best fit.

Furthermore, we observed the following based on the experimental results and the modelling exploration:
TABLE IV: Model parameters fit to measurement results without (OLS) and with (ML) consideration of censored data in different environments.

\begin{tabular}{|c|c|c|c|c|c|c|}
\hline \multirow{2}{*}{ Environment } & \multicolumn{2}{|c|}{$\hat{P L}\left(d_{0}\right)$} & \multicolumn{2}{|c|}{$\hat{n}$} & \multicolumn{2}{|c|}{$\hat{\sigma}$} \\
\hline & ML & OLS & ML & OLS & ML & OLS \\
\hline Urban & 74.85 & 60.06 & 2.75 & 3.20 & 11.25 & 10.84 \\
\hline Coastal & 43.96 & 18.33 & 3.62 & 4.24 & 27.51 & 22.81 \\
\hline Forested & 95.52 & 94.40 & 2.03 & 2.03 & 6.87 & 7.21 \\
\hline
\end{tabular}

- When using a distance-dependent variance, the model overfits the censored samples at short distances, yielding unrealistic parameters. We conclude that the distancedependency of the variance is insignificant. The CDF of the distances of unobserved samples shown in Figure 6illustrates that these were registered also at short distances.

- We have also performed the model fitting for the dualslope $(\mathrm{Eq} 3$ path loss model. The latter, in contrast to our initial expectation, did not provide a better matching. This is probably explained by the considerable amount of censored data we measured and took into account.

- The measurement procedure based on a handheld receiver, as presented in section III] provides a practical yet sub-optimal approach. We suspect that the position of the body of the person holding the device with respect to the LoS has impacted the observed PL significantly in particular measurements.

- No noticeable differences were observed between measurements conducted with the two types of antennas.

We provide specific interpretations of the results concerning the reliability of LoRa P2P links for the different environments below, considering the conditions in which the experiments were conducted. 
TABLE V: Root Mean Square Error and Mean Absolute Error of the considered models with respect to the obtained PL samples in Leuven.

\begin{tabular}{lll}
\hline Model & RMSE (dB) & MAE (dB) \\
\hline Our Urban Model (ML) & $\mathbf{1 1 . 0 1}$ & $\mathbf{8 . 9 7}$ \\
Our Urban Model (OLS) & 16.48 & 13.89 \\
Oulu Model (Car) [9] & 32.86 & 32.24 \\
Oulu Model (Boat) [9] & 29.99 & 29.44 \\
Okumura Hata Model & 69.89 & 68.25 \\
COST Hata Model & 35.72 & 32.44 \\
\hline
\end{tabular}

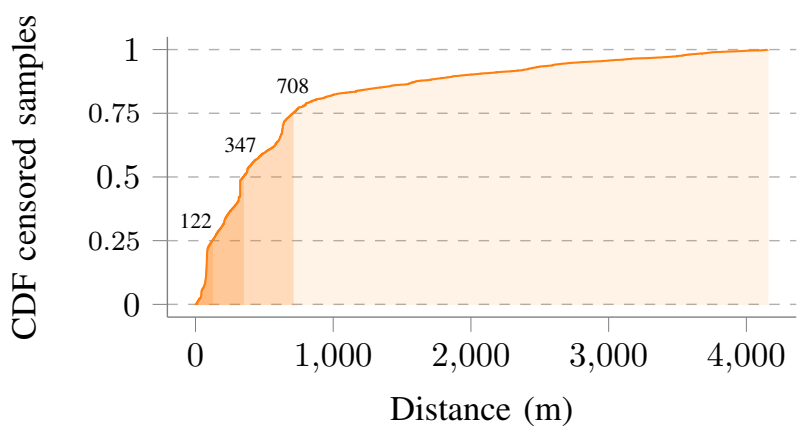

Fig. 6: Number of unobserved messages w.r.t. the $\mathrm{Rx}-\mathrm{Tx}$ separation. It clearly demonstrates that we already register censored samples at short distances $-25 \%$ of the censored data is present at distances lower than $122 \mathrm{~m}$.

\section{A. Reliability of Long Range Point-to-Point links}

The reliability of a connection depends, among other things, on the sensitivity of the receiver. Packets received below the demodulation floor are not captured by the receiver. To evaluate the reliability of Point-to-Point LoRa links, we consider the PER. We use the path loss estimated with our model $\left(P L_{\mathrm{m}}\right)$ to determine the probability of receiving packets below the demodulation floor. For instance, a reliability of minimum $84.2 \%$ is presumed up to the distance where the first $\sigma$-bound intersects with the path-loss threshold $\left(P L_{\mathrm{th}}\right)$.

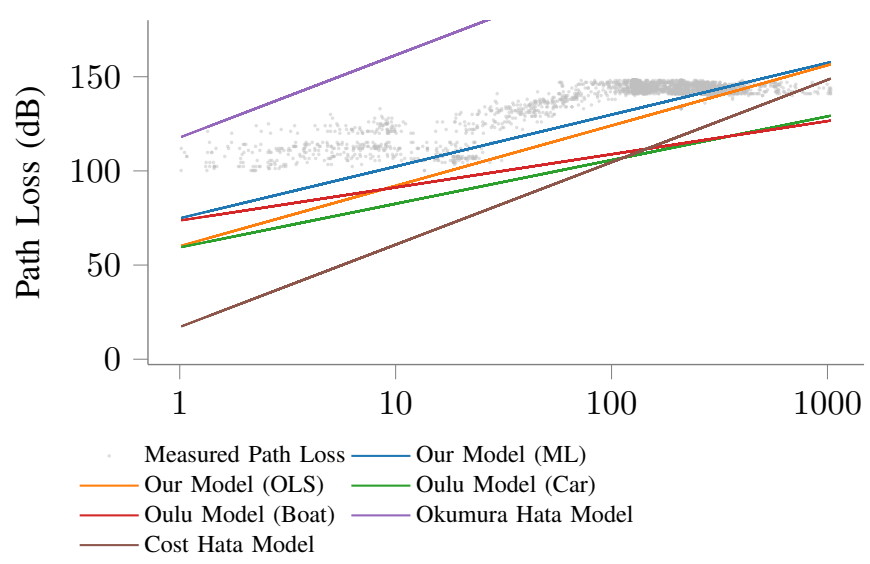

Fig. 7: Comparison of path loss models considering validation data obtained in an urban environment (Leuven). It demonstrates that our model outperforms the other models.
TABLE VI: Estimated single slope (Eq. 1p path loss parameters for each environment.

\begin{tabular}{lllll}
\hline Environment & weight & $P \hat{L\left(d_{0}\right)}$ & $\hat{n}$ & $\hat{\sigma}$ \\
\hline \multirow{4}{*}{ Urban } & No & 74.85 & 2.75 & 11.25 \\
& Linear & 66.57 & 3.17 & 14.93 \\
& Log10 & 73.65 & 2.81 & 11.92 \\
& Square & 68.92 & 3.06 & 11.96 \\
& & & & \\
Coastal & No & 43.96 & 3.62 & 27.51 \\
& Linear & 44.05 & 3.63 & 27.48 \\
& Log10 & 33.60 & 4.01 & 25.44 \\
& Square & 64.45 & 2.66 & 24.36 \\
\hline
\end{tabular}

Note that the $P L \hat{\left(d_{0}\right)}$ at the reference distance of $1 \mathrm{~m}$ is determined by the fitting method and does not necessarily reflect the experimental values.

The probability of receiving a packet below the demodulation floor with respect to the Rx-Tx separation distance is shown in Figure 8a In our experiments, we did not employ retransmissions. In actual data communication, these can improve the PER considerably, depending on the momentary load on the network [28]. Notably, the PER also depends on the SNR demodulation floor (Table I I). However, we have observed that a large fragment of packets are lost due to the RSS sensitivity opposed to a low SNR [29]. The modelled PER is also in line with the observed PER of the test data set as shown in Figure $8 \mathrm{a}$

\section{B. Service-range in Urban, Coastal and Forested Scenarios}

a) Urban Environment: Despite the unfavorable radio propagation conditions in the urban scenario, a maximum range of $1 \mathrm{~km}$ can still be achieved. Due to the obstructions present in the urban scenario, a relatively high PER is experienced. Neglecting the censored data points results in an overall lower PER w.r.t. the distance. This is illustrated in Figure $8 \mathrm{~b}$

b) Coastal Terrain: The coastal terrain clearly illustrates the favorable effect of a line-of-sight connection. The combination of line-of-sight and utilizing SF12 results in a coverage of over $4 \mathrm{~km}$; even with low-height terminals. Inspecting the matching of the PL models for the case for the coastal terrain, we observe that the path loss exponent is greatly overestimated in OLS. The relatively high variation around the mean large-scale fading $(\sigma)$ in the coastal environment results in a higher PER at shorter distances. We attribute this effect to the handheld device-based experimental campaign. Persons performing the measurements have walked around freely and were not asked to stay in the LoS between the Tx and Rx.

c) Experiments in the forest: As we were unable to collect RF samples inside the densily forested parts, the path loss is dominated by samples retrieved on the paths present in the forest. We have observed that the trees around the path ways behave as a waveguide yielding a path loss exponent close to the free space path loss exponent of 2 .

\section{Applications in the FiEld}

There is a vast diversity in the environments where it is desirable to deploy LPWAN-based IoT systems. Applications 


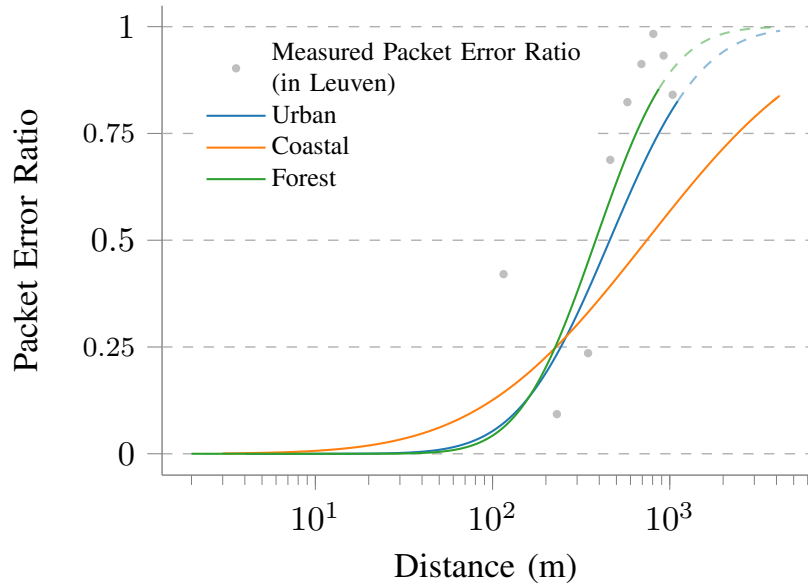

(a) PER for the three scenario including censored data points and without weighting. The dashed lines indicate the distances extending the coverage of the experiment.

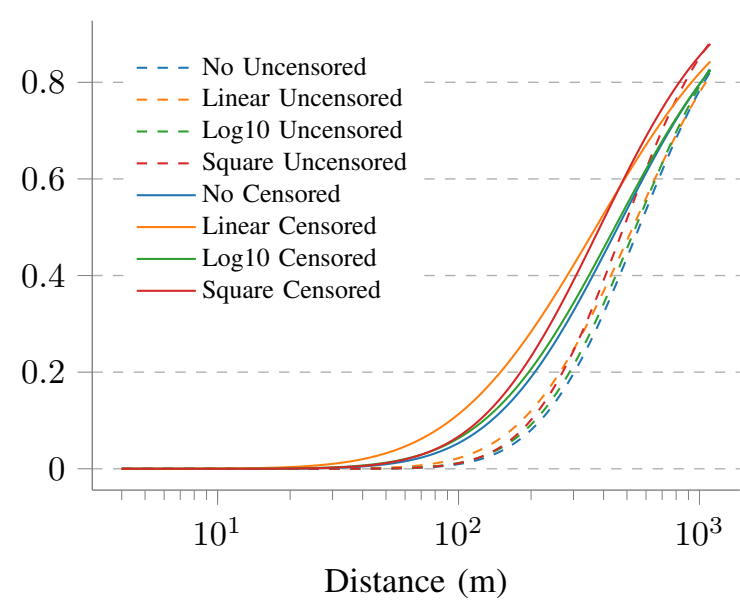

(b) PER for each weighting option for both the uncensored-only samples and all samples in the urban scenario. Neglecting the censored data points yields an underestimate of the PER at short distances.

Fig. 8: Packet error ratio for a LoRa Point-to-Point packet based on the estimated single slope models.

encountering diverse terrain and sites include (but are not limited to) monitoring trees in orchards [21], tracking location and health of wildlife or livestock (agriculture) [30], tracking shipping containers in harbors (logistics) and communicating with sensing buoys. As stated in [31], 26\% of drinking water of cows is lost due to leaking, resulting in lower milk production and, in some cases, even death. In order to address this issue, Mitchell and Gupta [31] developed low-cost flow sensors to detect leakage. The data was transmitted to a central processing unit with a $433 \mathrm{MHz}$ radio. An alternative wireless solution can be to employ the LoRa physical layer where devices communicate in a P2P fashion. And even in the case of very large farms, a multi-hop network could increase the range and reliability of the system. These topologies are expected to play a key role in projects such as Internet of Food \& Farming $4^{4}$ where Low-Power Wide-Area Network (LPWAN) low-cost, scalable and user-friendly solutions are vital.

\section{CONCLUSIONS}

We have assessed the path loss of point-to-point LoRa links with low-height terminals. We have built a movable experimental setup and made the design available in open source. With this setup we performed RSS channel measurements in urban, coastal and densely forested environments. A higher path loss is experienced and hence more packets are lost due to the low received signal strength compared to a typical star topology network with a base station at elevated height. Still, in all environments coverage of several tens of meters $(\sim 100 \mathrm{~m})$ to kilometers is achieved. These results confirm that LoRa is an interesting technology to establish ad-hoc wireless connectivity for many IoT applications. Note, that the range can be further extended by increasing the height of both nodes and creating a LoS connection. By doing so, we were able to receive packets at a distance of over $40 \mathrm{~km}^{5}$

4 www.iof2020.eu

5 http://dramco.be/measurements/over-the-horizon-iot/
Furthermore, we estimated path loss models for the considered environments. The maximum observable path loss is limited by the LoRa receiver sensitivity. When the unobserved samples are not considered, the estimated path loss parameters are hence biased. We applied the Tobit maximum-likelihood estimator to include censored samples in the estimation of the parameters of the path loss models. We observed an path loss exponent close to the free space exponent in the forested environment as the result of the alignment of trees acting as a waveguide. Other path loss models were compared to the obtained PL model and validated based on experimental data from an endorsing measurement campaign in an urban scenario. We show that the derived PL model based on ML outperforms employing the ordinary least squares approach and existing empirical models in the considered setting.

The path loss models fit to the characteristics of the different environments can be used in link simulators, aiding in the design of radio systems and deployment strategies. While the presented findings are a result of point-to-point measurements, they can also be applied in the design of LoRa mesh networks [20] to improve the energy efficiency and reliability over existing LoRa systems [32].

\section{ACKNOWLEDGMENT}

We thank our colleagues from the DRAMCO team for aiding in the measurement campaigns (with special efforts by Geoffrey Ottoy, Guus Leenders, Jarne Van Mulders, Chesney Buyle and Stijn Crul) and Fredrik Tufvesson for his valuable input.

\section{REFERENCES}

[1] LoRa Alliances. LoRaWAN 1.1 Specification. [Online]. Available: https://lora-alliance.org/sites/default/files/2018-04/ lorawantm_specification_-v1.1.pdf

[2] P. Jörke, S. Böcker, F. Liedmann, and C. Wietfeld, "Urban channel models for smart city IoT-networks based on empirical measurements of LoRa-links at 433 and $868 \mathrm{MHz}$," in 2017 
IEEE 28th Annual International Symposium on Personal, Indoor, and Mobile Radio Communications (PIMRC), Oct. 2017, pp. 1-6.

[3] D. Magrin, M. Centenaro, and L. Vangelista, "Performance evaluation of LoRa networks in a smart city scenario," in 2017 IEEE International Conference on Communications (ICC), May 2017, pp. 1-7.

[4] M. Lauridsen, H. Nguyen, B. Vejlgaard, I. Z. Kovacs, P. Mogensen, and M. Sorensen, "Coverage Comparison of GPRS, NB-IoT, LoRa, and SigFox in a 7800 kmš Area," in 2017 IEEE 85th Vehicular Technology Conference (VTC Spring), Jun. 2017, pp. 1-5.

[5] M. Aref and A. Sikora, "Free space range measurements with Semtech Lora technology," in 2014 2nd International Symposium on Wireless Systems within the Conferences on Intelligent Data Acquisition and Advanced Computing Systems, Sep. 2014, pp. 19-23.

[6] J. Petäjäjärvi, K. Mikhaylov, M. Hämäläinen, and J. Iinatti, "Evaluation of LoRa LPWAN technology for remote health and wellbeing monitoring," in 2016 10th International Symposium on Medical Information and Communication Technology (ISMICT), Mar. 2016, pp. 1-5.

[7] R. Sanchez-Iborra, J. Sanchez-Gomez, J. Ballesta-Viñas, M.D. Cano, and A. Skarmeta, "Performance evaluation of lora considering scenario conditions," Sensors, vol. 18, no. 3, p. 772, 2018.

[8] J. A. Gay-Fernández and I. Cuiñas, "Peer to Peer Wireless Propagation Measurements and Path-Loss Modeling in Vegetated Environments," IEEE Transactions on Antennas and Propagation, vol. 61, no. 6, pp. 3302-3311, Jun. 2013.

[9] J. Petajajarvi, K. Mikhaylov, A. Roivainen, T. Hanninen, and M. Pettissalo, "On the coverage of lpwans: range evaluation and channel attenuation model for lora technology," in 2015 14th International Conference on ITS Telecommunications (ITST), Dec 2015, pp. 55-59.

[10] L. Gregora, L. Vojtech, and M. Neruda, "Indoor signal propagation of LoRa technology," in 2016 17th International Conference on Mechatronics - Mechatronika (ME), Dec. 2016, pp. $1-4$.

[11] S. Hosseinzadeh, H. Larijani, K. Curtis, A. Wixted, and A. Amini, "Empirical propagation performance evaluation of LoRa for indoor environment," in 2017 IEEE 15th International Conference on Industrial Informatics (INDIN), Jul. 2017, pp. 26-31.

[12] M. R. Kielgast, A. C. Rasmussen, M. H. Laursen, J. J. Nielsen, P. Popovski, and R. Krigslund, "Estimation of received signal strength distribution for smart meters with biased measurement data set," IEEE Wireless Communications Letters, vol. 6, no. 1, pp. 2-5, 2017.

[13] C. Gustafson, T. Abbas, D. Bolin, and F. Tufvesson, "Tobit Maximum-likelihood estimation of Censored Pathloss Data," p. 9.

[14] A. Karttunen, C. Gustafson, A. F. Molisch, R. Wang, S. Hur, J. Zhang, and J. Park, "Path loss models with distancedependent weighted fitting and estimation of censored path loss data," IET Microwaves, Antennas Propagation, vol. 10, no. 14, pp. 1467-1474, 2016.

[15] B. Thoen, G. Callebaut, and G. Leenders, "Dramco UNO Low Power and Low Cost Arduino LoRa board." [Online]. Available: https://doi.org/10.5281/zenodo.2476999

[16] G. Callebaut, C. Buyle, and G. Leenders, "LoRa Point-to-Point Modules based on the Dramco UNO." [Online]. Available: https://doi.org/10.5281/zenodo.2660247

[17] G. Callebaut, "Path Loss Models for LoRa Point-to-Point Links." [Online]. Available: https://doi.org/10.5281/zenodo. 3407066

[18] "SX1276/77/78/79 - 137 MHz to 1020 MHz Low Power Long Range Transceiver." [Online]. Available: https://www.semtech. com/uploads/documents/DS_SX1276-7-8-9_W_APP_V5.pdf
[19] W. Shi, J. Cao, Q. Zhang, Y. Li, and L. Xu, "Edge Computing: Vision and Challenges," IEEE Internet of Things Journal, vol. 3, no. 5, pp. 637-646, Oct 2016.

[20] D. Lundell, A. Hedberg, C. Nyberg, and E. Fitzgerald, "A routing protocol for lora mesh networks," in 2018 IEEE 19th International Symposium on A World of Wireless, Mobile and Multimedia Networks (WoWMoM). IEEE, 2018, pp. 14-19.

[21] B. Thoen, G. Callebaut, G. Leenders, and S. Wielandt. Internetof-Trees. [Online]. Available: https://dramco.be/projects/iotree/

[22] M. N. Ochoa, A. Guizar, M. Maman, and A. Duda, "Evaluating lora energy efficiency for adaptive networks: From star to mesh topologies," in 2017 IEEE 13th International Conference on Wireless and Mobile Computing, Networking and Communications (WiMob), Oct 2017, pp. 1-8.

[23] Siretta. Delta 11. [Online]. Available: https://www.siretta.co. uk/download.php?id=648\&cmd=view

[24] Taoglas. PC81.07.0100A.db. [Online]. Available: https://cdn. taoglas.com/datasheets/PC81.07.0100A.db.pdf

[25] A. Molisch, Wireless Communications. Wiley-IEEE Press, 2005.

[26] C. Gustafson, T. Abbas, D. Bolin, and F. Tufvesson, "Statistical Modeling and Estimation of Censored Pathloss Data," IEEE Wireless Communications Letters, vol. 4, no. 5, pp. 569-572, Oct 2015.

[27] J. Tobin, "Estimation of relationships for limited dependent variables," Econometrica, vol. 26, no. 1, pp. 24-36, 1958. [Online]. Available: http://www.jstor.org/stable/1907382

[28] G. Callebaut, G. Ottoy, and L. Van der Perre, "Cross-Layer Framework and Optimization for Efficient Use of the Energy Budget of IoT Nodes," in 2019 IEEE Wireless Communications and Networking Conference (WCNC), 2019.

[29] G. Callebaut, G. Leenders, C. Buyle, S. Crul, and L. V. der Perre, "LoRa Physical Layer Evaluation for Point-to-Point Links and Coverage Measurements in Diverse Environments," in 2019 European Conference on Networks and Communications (EuCNC), 2019.

[30] E. D. Ayele, N. Meratnia, and P. J. Havinga, "MANER: Managed data dissemination scheme for LoRa IoT enabled wildlife monitoring system (WMS)," 2018 9th IFIP International Conference on New Technologies, Mobility and Security, NTMS 2018 - Proceedings, vol. 2018-Janua, pp. 1-7, 2018.

[31] C. Mitchell and G. S. Gupta, "Techniques for Autonomous Water Fault Detection on Large Dairy Farms," in 2019 IEEE Sensors Applications Symposium (SAS), 2019.

[32] H. Lee and K. Ke, "Monitoring of large-area iot sensors using a lora wireless mesh network system: Design and evaluation," IEEE Transactions on Instrumentation and Measurement, vol. 67, no. 9, pp. 2177-2187, Sep. 2018.

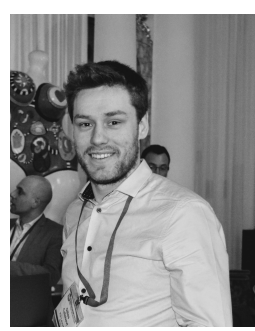

Gilles Callebaut is pursuing a $\mathrm{Ph} . \mathrm{D}$. in Massive MIMO for low power Machine Type Communication (MTC). He initiated the tutorial "Low Power Wireless Technologies for Connecting Embedded Sensors in the IoT: A Journey from Fundamentals to Hands-on". Gilles graduated summa cum laude in 2016 and received the M.Sc. degree in engineering technology at KU Leuven campus Ghent, Belgium. In addition, he received the laureate award. $\mathrm{He}$ is currently a member of DRAMCO, a research group which is focused on wireless and mobile communication systems. His main interests are Machine Type Communication (MTC), Internet of Things (IoT), low power embedded systems and everything mobile. 


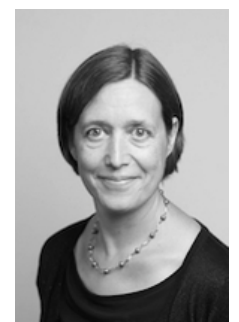

Liesbet Van der Perre is Professor at the department of Electrical Engineering at the KU Leuven in Leuven, Belgium and a guest Professor at the Electrical and Information Technology Department at Lund University, Sweden. Dr. Van der Perre was with the nano-electronics research institute imec in Belgium from 1997 till 2015, where she took up responsibilities as senior researcher, system architect, project leader and program director. Dr. Van der Perre's main research interests are in wireless communication, with a focus on physical layer and energy efficiency in both broadband communication and IoT. She is an author and co-author of over 300 scientific publications. She was appointed honorary doctor at Lund University, Sweden, in 2015. She received her Masters and a $\mathrm{Ph} . \mathrm{D}$. degree from the KU Leuven, Belgium, in 1992 and 97 respectively. 